\title{
From solid to granular gases: the steady state for granular materials
}

\author{
D. Vescovi ${ }^{1 *}$, C. di Prisco ${ }^{2}$, D. Berzi ${ }^{1}$ \\ ${ }^{1}$ Dept. of Environmental, Hydraulic, Infrastructure, and Surveying Engineering, Politecnico di Milano, Milan, Italy. \\ ${ }^{2}$ Dept. of Structural Engineering, Politecnico di Milano, Milan, Italy.
}

\begin{abstract}
SUMMARY
This paper aims at extending the well known critical state concept, associated with quasi-static conditions, by accounting for the role played by the strain rate when focusing on the steady, simple shear flow of a dry assembly of identical, inelastic, soft spheres. An additional state variable for the system, the granular temperature, is accounted for. The granular temperature is related to the particle velocity fluctuations and measures the agitation of the system. This state variable, as is in the context of kinetic theories of granular gases, is assumed to govern the response of the material at large strain rates and low concentrations.

The stresses of the system are associated with enduring, frictional contacts among particles involved in force chains and nearly instantaneous collisions. When the first mechanism prevails, the material behaves like a solid, and constitutive models of soil mechanics hold; whereas when inelastic collisions dominate, the material flows like a granular gas and kinetic theories apply. Considering a pressure-imposed flow, at large values of the normal stress and small values of the shear rate, the theory predicts a non monotonic behaviour of the stress ratio at the steady state, which is likely to govern the evolution of landslides. Copyright (c) 0000 John Wiley \& Sons, Ltd.

Received ...
\end{abstract}

KEY WORDS: constitutive modeling; granular flows; steady state; theoretical analysis; simple shear

\section{INTRODUCTION}

Since the pioneering works of Roscoe et al.[1] and Schofield and Wroth [2], the critical state concept in soil mechanics has been introduced as a limit state condition which is reached by means of evolving loading paths. Experimental investigations,especially using strain controlled triaxial tests, have been performed to describe such an ideal condition, as well as many constitutive models have been conceived by starting from such a theoretical definition [3, 4]. On the contrary, the most of works published within the granular flow community (e.g., see [5]) deals with the rheology of granular materials at large strain rates and low to moderate concentration, far from the quasi-static

\footnotetext{
${ }^{*}$ Correspondence to: D. Vescovi, Dept. of Environmental, Hydraulic, Infrastructure, and Surveying Engineering, Politecnico di Milano, 20133 Milan, Italy. E-mail: dalila.vescovi@mail.polimi.it

Copyright (c) 0000 John Wiley \& Sons, Ltd.

Prepared using nagauth.cls [Version: 2010/05/13 v2.00]
} 
conditions, and prevalently considers simple shear tests (rheometers) under steady conditions. Within this community, kinetic theories [6, 7, 5], according to which the inelastic collisions associated with the random motion of the grains represent the main mechanism to dissipate the energy of the system, have been developed. The theories take into consideration granular gaseous or collisional states, in which force chains within the medium, forming the granular skeleton, disappear.

There are several practical problems where the granular material encompasses a transition from a solid to a more gaseous state, thus suggesting that a collaboration between the two above mentioned communities would be fruitful. The landslide risk evaluation, requiring the modeling of both the inception and the evolution of the gravitational collapse, is the tipical example. Moreover, the increasing success of computational tools in handling large deformations suggests that such an ambitious goal is now possible and stimulates the need for constitutive models capable of simulating the mechanical response of granular materials under both quasi-static and collisional conditions. The first step in this direction is the extension of the critical state concept, interpreted hereafter as a sort of limit condition for the steady state at vanishingly small strain rate, by employing the granular temperature as in [8], as an additional state variable for the system. When the granular temperature, $T$, defined as the mean square of the velocity fluctuations, quantitatively describing the degree of agitation of the system, is large, the stored energy of the system is prevalently kinetic; whereas, at small $T$, the stored energy of the system is mainly elastic. In this perspective, a recent constitutive model [9] for the granular material, valid under both quasi-static and collisional conditions, is slightly modified, mechanically interpreted in the light of visco-plasticity and parametrically discussed.

This paper wants to suggest a road map, allowing the Geotechnicians to get outside from their "one-dimensional world" and discovering, as for the inhabitants of Flatland in the well-known masterpiece of Abbott [10], the "marvelous" multi-dimensional state-variable universe.

\section{THEORY}

The Geotechnical community usually associates the concept of critical state with a non-evolving state reached after a progressive increase in strain, at a vanishingly small strain-rate. At the critical state, an ideal mechanism of yielding is assumed to develop within the specimen: the external work is totally dissipated by frictional processes at the contact level (disregarding both crushing and damage); the micro-structure does not evolve and, consequently, the void ratio, $e$, remains constant (i.e., segregation is inhibited). Focusing on triaxial tests (recently also by means of distinct element numerical simulations [11]), Geotechnicians have traditionally defined the locus of the critical state using the void ratio, the effective pressure and the deviator (defined as the difference between the axial and radial stress). Conversely, only few works have dealt with simple shear flows (Fig. 1), which instead represents the most studied configuration in determining the rheology of granular gases [12, 13, 14, 15]. 


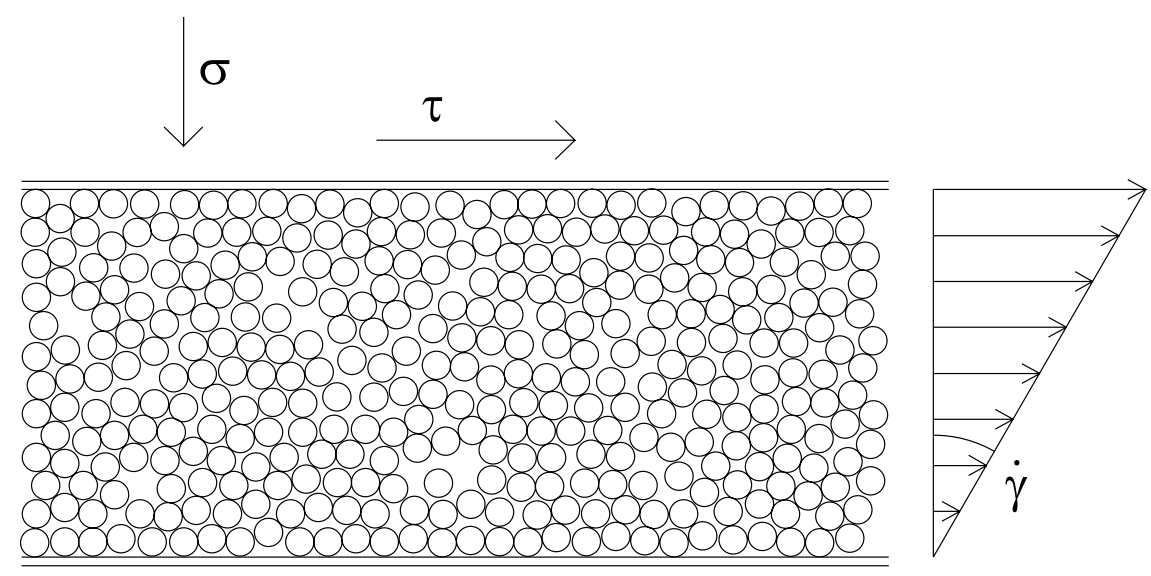

Figure 1. Simple shear flow configuration.

Limiting the analysis, for the sake of simplicity, on the homogeneous simple shear of an assembly of identical, dry spherical particles of diameter $d$ and density $\rho_{p}$, the variables governing the problem are the shear stress $\tau$, the normal stress along the transversal direction, $\sigma$, the void ratio, $e$, or alternatively, the concentration $\nu=1 /(1+e)$, the shear strain, the strain rate $\dot{\gamma}$ and the granular temperature $T$. In the realm of Geotechnique, the strain rate is usually taken to be zero, and therefore disregarded, whereas the granular temperature is ignored. In the realm of granular flows, the shear strain is infinite, as for classic fluids, therefore not influencing the problem. In our view, the two realms are strictly connected, and the critical state, for which the shear strain is infinite and both the strain rate and the granular temperature are zero, represents the boundary between them.

To merge the two approaches, it is quite useful considering the energy balance of the system. In the simple shear flow, at the steady state, under the usual assumptions of constant shear and normal stresses, the flux of energy is neglected, so that the energy created by the work of the internal stresses equals the dissipated energy. $\Gamma_{\mathrm{q}}$ being the rate at which energy is dissipated in frictional, enduring contacts and $\Gamma_{\mathrm{c}}$ the energy dissipated by collisions, the balance of energy for the simple, shear flow reduces to

$$
\tau \dot{\gamma}=\Gamma_{\mathrm{q}}+\Gamma_{\mathrm{c}} .
$$

As suggested by several authors [16, 17, 18, 19, 9], we assume to subdivide the granular stresses into two contributions:

$$
\begin{aligned}
& \sigma=\sigma_{\mathrm{q}}+\sigma_{\mathrm{c}}, \\
& \tau=\tau_{\mathrm{q}}+\tau_{\mathrm{c}} .
\end{aligned}
$$

Here and in what follows, the subscript $q$ (quasi-static) and $c$ (collisional) refer to quantities associated with enduring, frictional contacts of particles involved in force chains (soil skeleton) and nearly instantaneous collisions, respectively. By substituting Eq. (2) into Eq. (1), we obtain

$$
\tau_{\mathrm{q}} \dot{\gamma}+\tau_{\mathrm{c}} \dot{\gamma}=\Gamma_{\mathrm{q}}+\Gamma_{\mathrm{c}}
$$


Unlike suggested in other works [19], enduring contacts among particles in force chains cannot produce fluctuating energy. As a consequence, Eq. (3) reduces to

$$
\begin{aligned}
\tau_{\mathrm{q}} \dot{\gamma} & =\Gamma_{\mathrm{q}}, \\
\tau_{\mathrm{c}} \dot{\gamma} & =\Gamma_{\mathrm{c}} .
\end{aligned}
$$

\subsection{Quasi-static contribution}

The quasi-static component of the shear stress is assumed to be proportional to the quasi-static component of the normal stress through the tangent of the internal friction angle at the critical state $\phi_{s s}^{\prime},[2,20]:$

$$
\tau_{\mathrm{q}}=\sigma_{\mathrm{q}} \tan \phi_{s s}^{\prime} .
$$

$\phi_{s s}^{\prime}$ being a function of both the inter-particle friction coefficient $\phi_{\mu}$ and the simple shear constraints [21].

For dimensional reasons, the critical state locus in the $\sigma-\nu$ plane can be written as

$$
\sigma_{\mathrm{q}}=f_{0} \frac{K}{d}
$$

where the particle stiffness $K$ is equal to $\pi d E / 8$ in the case of linear elastic contacts [22], with $E$ is the Young's modulus, whereas $f_{0}$ is solely a function of the concentration.

To be consitent with physical observations on granular packings, we assume that the function $f_{0}$ vanishes when the concentration is equal to the random loose packing value, $\nu_{\text {rlp }}$, defined as the minimum concentration at which a disordered packing exists [23]. In other words, at the random loose packing, the granular material undergoes a phase transition to a purely collisional regime. The concentration at random loose packing is a decreasing function of the inter-particle friction coefficient $\phi_{\mu}[23,24,25]$. For frictionless particles, that is, $\phi_{\mu}=0, \nu_{\mathrm{rlp}}$ coincides with the random close packing, $\nu_{\text {rcp }}=0.636$, defined as the densest possible disordered packing of identical spheres [23]. On the other hand, $f_{0}$ must diverge at $\nu_{\mathrm{s}}$, another critical value, at which the force chains span the entire domain and a shear rigidity develops [26]. Therefore, we take

$$
f_{0}= \begin{cases}a \frac{\nu-\nu_{\mathrm{rlp}}}{\nu_{\mathrm{S}}-\nu} & \text { if and only if } \nu>\nu_{\mathrm{rlp}} \text { and } \nu_{\mathrm{rlp}}<\nu_{\mathrm{S}} \\ 0 & \text { otherwise }\end{cases}
$$

where $a$ is a dimensionless material coefficient [9].

Experimental investigations on the critical state of identical spheres are though rare. To our knowledge, only Wroth [27] performed experiments on the critical state of $1 \mathrm{~mm}$ stainless steel spheres $\left(K=8.25 \cdot 10^{7} \mathrm{~Pa} \mathrm{~m}\right)$ using a shear cell [28]. The experiments confirm that the ratio of $\tau_{\mathrm{q}}$ to $\sigma_{\mathrm{q}}$ is constant and that $f_{0}$ is a unique function of the concentration. In Fig. 2(a) the theoretical expression of Eq. (7), with $\nu_{\mathrm{s}}=0.619, \nu_{\mathrm{rlp}}=0.598$ and $a=1.8 \cdot 10^{-6}$, obtained from linear regression, is drawn. The data of Fig. 2(a) are plotted in terms of $f_{0}$ against void ratio in Fig. 2(b), 


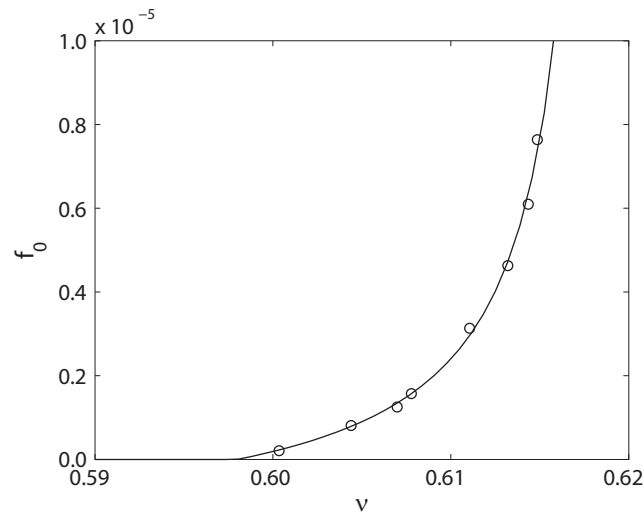

(a)

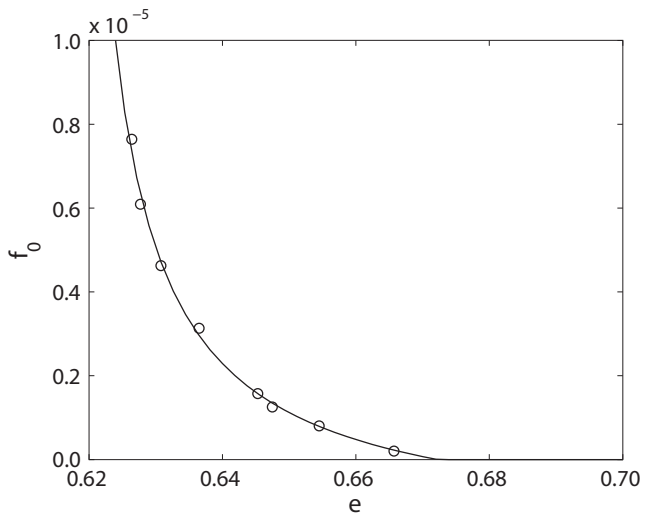

(b)

Figure 2. Experimental (circles, after [27]) and theoretical (solid line) coefficient $f_{0}$ for steel spheres as function of (a) concentration and (b) void ratio.

\subsection{Collisional contribution}

The constitutive relations for the collisional stresses and the rate of dissipation of fluctuating energy are those proposed by Garzó and Dufty [29], as modified by Jenkins and Berzi [26],

$$
\begin{gathered}
\sigma_{\mathrm{c}}=\rho_{p} f_{1} f_{4} T, \\
\tau_{\mathrm{c}}=\rho_{p} d f_{2} f_{4} T^{1 / 2} \dot{\gamma},
\end{gathered}
$$

and

$$
\Gamma_{\mathrm{c}}=\rho_{p} \frac{f_{3}}{L} f_{4} T^{3 / 2}
$$

Functions $f_{1}, f_{2}$ and $f_{3}$, reported in Tab. I] are solely dependent on the concentration $\nu$ and $\epsilon$. The latter is an effective coefficient of restitution which depends on (i) the normal coefficient of restitution (ratio of precollisional to postcollisional relative velocity between colliding particles in the normal impact direction), (ii) the tangential coefficient of restitution in a sticking collision, and (iii) the Coulomb friction coefficient characterizing sliding collisions [30]. In the elastic limit, that is, when $\epsilon=1, \nu_{\mathrm{s}}$ equals $\nu_{\mathrm{rcp}}$ [31]. In Eq. [10), $L$ is the correlation length, accounting for the decrease in the rate of collisional energy dissipation due to the correlated motion of particles that is likely to occur when the flow is dense [32, 33, 26]. In its expression, reported in Tab. [, $c$ is a dimensionless material coefficient of order unity.

The function $f_{4}$, not present in the constitutive relations of Jenkins and Berzi [26], takes into account the influence of the particle stiffness on the collisions, and explicitly depends on both the concentration and the granular temperature. By following [34],

$$
f_{4}=\left[1+2 \frac{d}{s}\left(\frac{\rho_{p} T}{E}\right)^{1 / 2}\right]^{-1},
$$

where $s$ is the mean separation distance among particles. At equilibrium, the latter can be identified as the mean free path (mean distance traveled by a particle between two successive collisions). In 
Table I. List of expressions for the collisional contribution to the stresses.

$$
\begin{aligned}
& \hline f_{1}=4 \nu G F \\
& f_{2}=\frac{8 J}{5 \pi^{1 / 2}} \nu G \\
& f_{3}=\frac{12}{\pi^{1 / 2}}\left(1-\epsilon^{2}\right) \nu G \\
& G=\nu g_{0} \\
& g_{0}= \begin{cases}\frac{(2-\nu)}{2(1-\nu)^{3}} \quad \nu \leq 0.49 \\
\frac{5.69\left(\nu_{\mathrm{S}}-0.49\right)}{\nu_{\mathrm{S}}-\nu} & \nu>0.49\end{cases} \\
& F=\frac{1+\epsilon}{2}+\frac{1}{4 G} \quad \frac{1+\epsilon}{2}+\frac{\pi}{32} \frac{[5+2(1+\epsilon)(3 \epsilon-1) G][5+4(1+\epsilon) G]}{\left[24-6(1-\epsilon)^{2}-5\left(1-\epsilon^{2}\right)\right] G^{2}} \\
& J=\max \left[1,\left(\frac{1}{2} c \frac{G^{1 / 3}}{T^{1 / 2}} d \dot{\gamma}\right)\right]
\end{aligned}
$$

the context of classic kinetic theories [35],

$$
s=\frac{\sqrt{2}}{12} \frac{d}{G} .
$$

By using Eqs. (2), (6) and (8),

$$
T=\frac{\sigma-f_{0} K / d}{\rho_{p} f_{1} f_{4}} .
$$

And also, from Eq. (13) and Eq. (11),

$$
\frac{1}{f_{4}}-2 \frac{d}{s} \sqrt{\frac{\pi}{8 f_{1}}\left(\frac{\sigma d}{K}-f_{0}\right)} \frac{1}{\sqrt{f_{4}}}-1=0
$$

that gives

$$
f_{4}=\frac{2}{2+\mathcal{A}+\sqrt{\mathcal{A}^{2}+4 \mathcal{A}}}
$$

where

$$
\mathcal{A}=\frac{36 \pi G^{2}}{f_{1}}\left(\frac{\sigma d}{K}-f_{0}\right) .
$$

As expected, $f_{4}$ tends to one as $K$ tends to infinity.

It is important to notice that taking into account the role of particle stiffness on the duration of a collision, the collisional contributions $\sigma_{\mathrm{c}}, \tau_{\mathrm{c}}$ and $\Gamma_{\mathrm{c}}$ depend not only on the concentration, the granular temperature and the shear rate, as in classic kinetic theories, but also on the ratio between the normal stress and the particle stiffness $\sigma /(K / d)$. 


\subsection{Constitutive relationship}

By substituting Eqs. (9) and (10) into (4), and using the constitutive expression for $L$ of Tab. I, the granular temperature results an algebraic function of the shear rate,

$$
T=d^{2} f_{5} \dot{\gamma}^{2}
$$

with

$$
f_{5}=\frac{L}{d} \frac{f_{2}}{f_{3}}
$$

and

$$
\frac{L}{d}=\max \left[1,\left(\frac{c^{2} G^{2 / 3} f_{3}}{4 f_{2}}\right)^{1 / 3}\right] .
$$

By introducing Eq. (17) into Eqs. (8) and (9), the expressions for the total stresses in steady, simple shear flows read

$$
\left\{\begin{array}{l}
\sigma=\frac{K}{d} f_{0}+\rho_{p} d^{2} f_{1} f_{4} f_{5} \dot{\gamma}^{2} \\
\tau=\frac{K}{d} f_{0} \tan \phi_{s s}^{\prime}+\rho_{p} d^{2} f_{2} f_{4} f_{5}^{1 / 2} \dot{\gamma}^{2}
\end{array}\right.
$$

Eqs. (20) represent an extension in a four dimensional space of the critical state condition to nonzero values of $\dot{\gamma}$ (or $T$ ). A graphycal illustration of such a locus is reported in Fig. 3, where the different lines in the $\tau-\sigma-e$ space correspond to different values of $T$. The material parameter values employed to obtain the curves coincide with those in Sect 3.2 An alternative way of writing

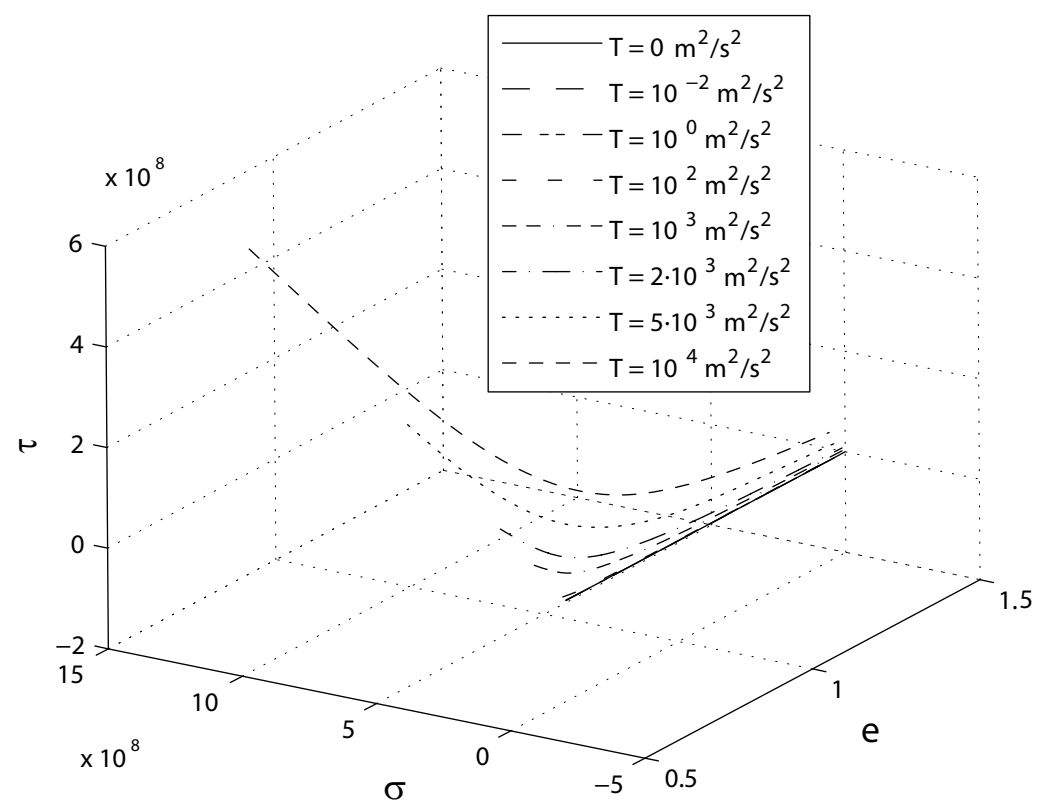

Figure 3. Evolution of the steady state locus as a function of the granular temperature $T$. 
Eqs. (20) is the following:

$$
\left\{\begin{array}{l}
1-\frac{K}{\sigma d} f_{0}-\frac{\gamma_{1}^{2}}{\gamma_{2}^{2}}\left(\frac{\tau}{\sigma}-\tan \phi_{s s}^{\prime}\right)=0 \\
\frac{\tau}{\sigma}-\tan \phi_{s s}^{\prime}-\frac{t_{m}^{2}}{\gamma_{1}^{2}} \dot{\gamma}^{2}=0
\end{array}\right.
$$

where $t_{m}=d\left(\rho_{p} \nu / \sigma\right)^{1 / 2}$ is the microscopic time scale associated with the rearrangement of particles [13], and

$$
\begin{aligned}
& \gamma_{1}=\left[\frac{\nu}{f_{4}\left(f_{2} f_{5}^{1 / 2}-\tan \phi_{s s}^{\prime} f_{1} f_{5}\right)}\right]^{1 / 2}, \\
& \gamma_{2}=\left[\frac{\nu}{f_{1} f_{4} f_{5}}\right]^{1 / 2}
\end{aligned}
$$

Eq. (21b) provides

$$
\dot{\gamma}=\frac{\gamma_{1}}{t_{m}}\left(\frac{\tau}{\sigma}-\tan \phi_{s s}^{\prime}\right)^{1 / 2},
$$

that can be interpreted, in the visco-plastic framework, as

$$
\dot{\gamma}=\tilde{\gamma} \Phi(\mathcal{F})
$$

where $\Phi(\mathcal{F})$ is the viscous nucleus function of the yield locus $\mathcal{F}$ [36]. In this case,

$$
\Phi(\mathcal{F})=(\mathcal{F})^{1 / 2}
$$

and

$$
\mathcal{F}=\frac{\tau}{\sigma}-\tan \phi_{s s}^{\prime}
$$

In Eq. 24 $\tilde{\gamma}$ is the fluidity parameter,

$$
\tilde{\gamma}=\frac{\gamma_{1}}{t_{m}}
$$

that is not constant, unlike commonly assumed in the literature.

The model parameters which affect the constitutive relations (21) can be subdivided into (i) micro-mechanical parameters, characteristics of the single particle (i.e., $\rho_{p}, d, K, \phi_{\mu}$ and $\epsilon$ ); (ii) macro-mechanical parameters, characteristics of the "continuum" medium (i.e., $\nu_{\mathrm{rl}}, \nu_{\mathrm{s}}, \tan \phi_{s s}^{\prime}$, $a$ and $c$ ). As previously mentioned, micro and macro-mechanical parameters are related to each other; for instance, the inter-particle friction coefficient affects the concentration at random loose packing and the critical friction angle, and the coefficient of collisional restitution influences the concentration at which the shear rigidity develops. Also $\epsilon$ and $\phi_{\mu}$ are not, in principle, totally independent. 


\section{DISCUSSION AND RESULTS}

In this section, the aforementioned constitutive relationship is theoretically discussed by (i) introducing a phase diagram, (ii) commenting the role played by the normal stress for pressureimposed flows, (iii) highlighting the occurence of a non-monotonic behaviour of the stress ratio for large values of $\sigma$ and (iv) solving the relationship for (iv-a) concentration and (iv-b) stress ratioimposed flows.

\subsection{Phase diagram}

From (20a), because the second term on the right hand side is always positive, it must be that

$$
1-\frac{K}{d \sigma} f_{0} \geq 0
$$

By substituting Eq. (7) into Eq. (28), for positive values of $f_{0}$, we obtain

$$
\nu \leq \nu_{\mathrm{m}}
$$

where

$$
\nu_{\mathrm{m}}=\frac{a \nu_{\mathrm{rlp}}}{a+\sigma d / K}+\frac{\nu_{\mathrm{s}}}{1+a(\sigma d / K)^{-1}}
$$

represents the maximum concentration that can be achieved under steady conditions, for a fixed value of $\sigma$, when the shear rate vanishes.

Fig. 4 shows the qualitative phase diagram in the plane concentration-normal stress. For large values of $\sigma d / K, \nu_{\mathrm{m}}$ approaches $\nu_{\mathrm{s}}$; on the other hand, $\nu_{\mathrm{m}}$ tends to $\nu_{\mathrm{rlp}}$ when $\sigma d / K$ is sufficiently small. Then, the range of coexistence of quasi-static and collisional stresses, said "transitional regime", depends on the imposed normal stress.

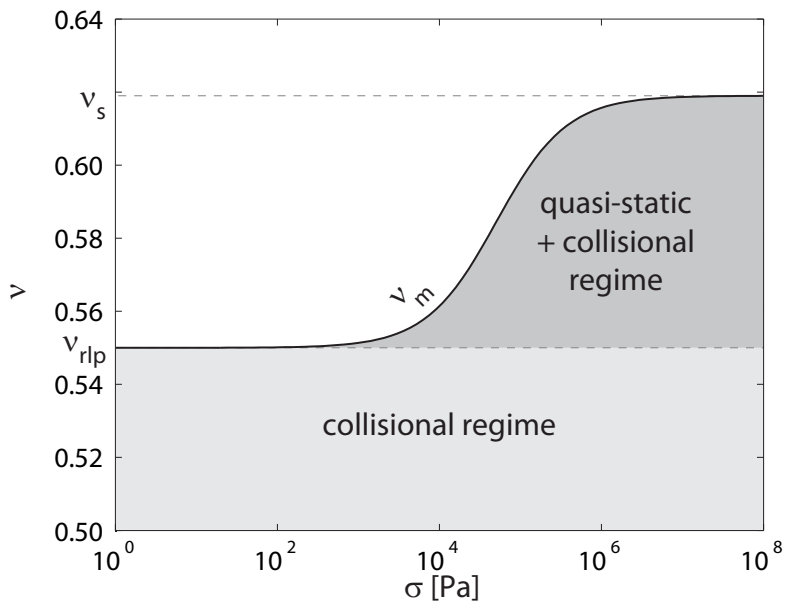

Figure 4. Phase diagram for steady, simple shear flow of inelastic spheres in the plane $\sigma-\nu$.

Analogously, in Fig. 51the qualitative phase diagram in the plane $\phi_{\mu}-\nu$ for the steady, simple shear flow of inelastic spheres, that is, $\epsilon<1$ and $\nu_{\mathrm{S}}<\nu_{\mathrm{rcp}}$, for an imposed value of the normal stress, is 
illustrated.

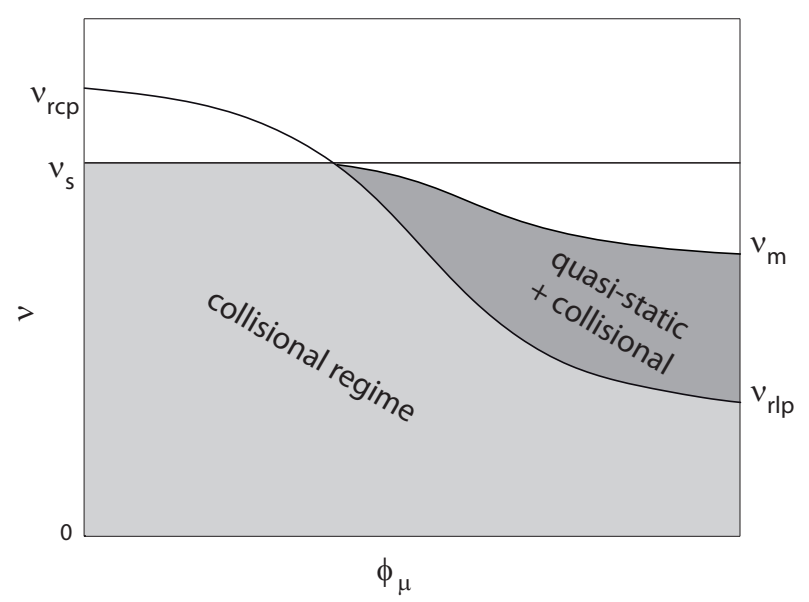

Figure 5. Phase diagram for the steady, simple shear flow of inelastic spheres at imposed value of $\sigma$.

For a given value of the inter-particle friction $\phi_{\mu}$, the concentration decreases as the shear rate increases (as already mentioned, the maximum value is when $\dot{\gamma}=0$, i.e., at the critical state, when the collisional stresses vanish). For small values of $\phi_{\mu}, \nu_{\mathrm{rlp}}$ is greater than $\nu_{\mathrm{S}}$ (here assumed to be constant, in absence of clear evidences of its possible dependence on $\phi_{\mu}$ ), so that the quasi-static stresses are zero: the maximum concentration therefore coincides with $\nu_{\mathrm{s}}$ and the steady, simple shear flow is always in the collisional regime. At larger $\phi_{\mu}, \nu_{\mathrm{rlp}}$ is lower than $\nu_{\mathrm{s}}$ : the concentration at the critical state is $\nu_{\mathrm{m}}$, and quasi-static and collisional stresses coexist in the range between $\nu_{\mathrm{rlp}} \leq \nu \leq \nu_{\mathrm{m}}$. At the value of $\dot{\gamma}$ which corresponds to a concentration equal to $\nu_{\mathrm{rlp}}$, the quasi-static stresses vanish and the material undergoes a phase transition to the collisional regime. The range of coexistence of quasi-static and collisional stresses depends on the ratio $\sigma d / K$, which affects the value of $\nu_{\mathrm{m}}$. In particular, for small values of $\sigma d / K$, i.e., small values of the total normal stress or large values of the particle stiffness, $\nu_{\mathrm{m}}$ approaches $\nu_{\mathrm{s}}$, as already mentioned, thus reducing the range of influence of quasi-static stresses on the flow.

\subsection{Pressure-imposed flow}

Steady, simple shear flows can be physically and numerically simulated (i) by imposing the normal stress, and measuring the concentration (or alternatively the void ratio) and the shear stress as functions of the shear rate (pressure-imposed); (ii) by imposing the concentration (void ratio), and measuring the normal and shear stress as functions of the shear rate (concentration-imposed); (iii) by imposing the stress ratio, and measuring the concentration and the shear rate as functions of the normal stress (stress ratio-imposed). The results of the three configurations are equivalent, if dimensionless quantities are employed [14]. In this section, we will consider pressure-imposed flows, commonly taking into consideration in the Geotechnical community, which can be considered as a mixed-control test.

All the results illustrated in Fig. 6refer to an ideal granular material which should somehow mimic 
the behaviour of sand. Hence, we take: $d=1 \mathrm{~mm} ; \rho_{p}=2600 \mathrm{~kg} / \mathrm{m}^{3} ; K=2.8 \cdot 10^{4} \mathrm{kPa} \mathrm{m}$ (from the Young's modulus for quartz); $\epsilon=0.6$ and $c=0.5$ (as appropriated for glass spheres, [26]); $a=1.8 \cdot 10^{-6}$, and $\nu_{\mathrm{s}}=0.619$ (from Wroth's experiments on stainless steel spheres, see section 2.1); $\tan \phi_{s s}^{\prime}=0.5$ (the tangent of the angle of repose obtained by Forterre and Pouliquen [37] for $0.8 \mathrm{~mm}$ sand); $\nu_{\mathrm{rlp}}=0.55$ (as appropriated for very frictional particles, [24]). Fig. 6(a) and 6(b) show respectively the stress ratio and the concentration as a function of the shear rate for four different values of the imposed normal stress.

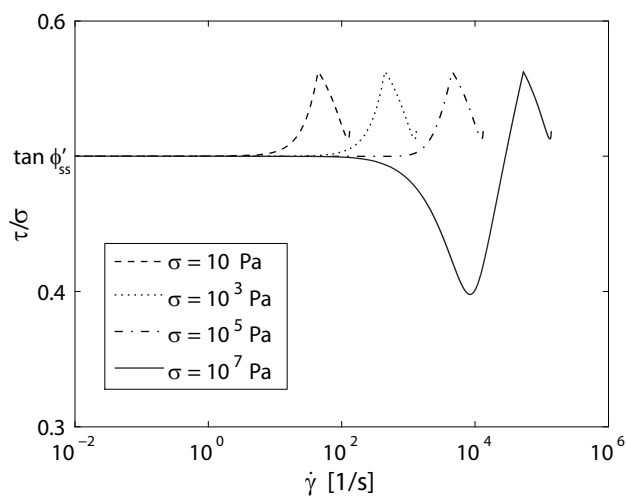

(a)

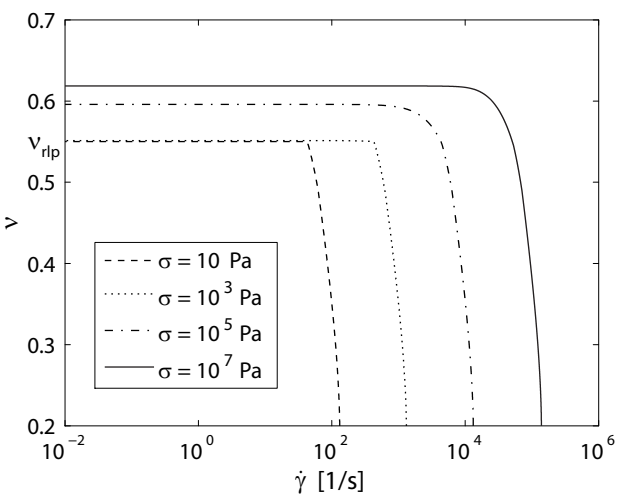

(b)

Figure 6. Theoretical (a) stress ratio and (b) concentration as a function of the shear rate for $1 \mathrm{~mm}$ sand, at different values of the applied normal stress.

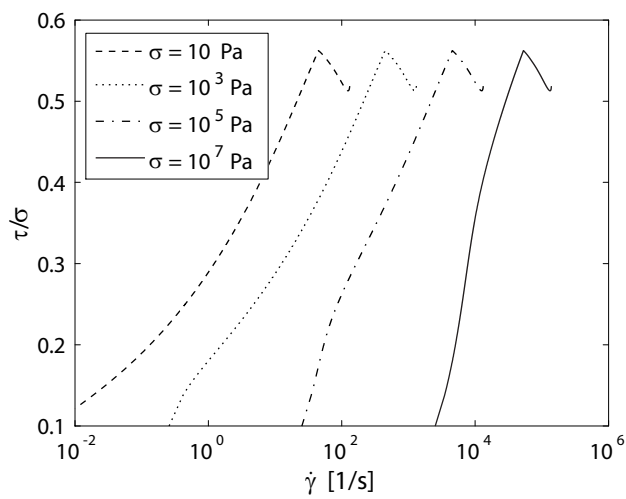

(a)

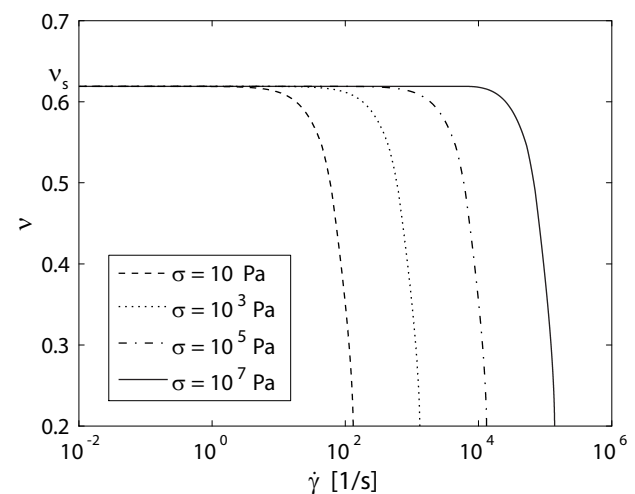

(b)

Figure 7. Theoretical (a) stress ratio and (b) concentration as a function of the shear rate for $1 \mathrm{~mm}$ sand, at different values of the applied normal stress, when quasi-static stresses are ignored (purely collisional model).

Fig. 6a shows that, in purely collisional regime, when $\tau / \sigma$ reaches the maximum value (0.562), all the curves exhibit a peak. The subsequent deacreasing behaviour in the purely collisional regime seems to be confirmed by numerical simulations on unbounded shear flows [15]. If the applied normal stress is sufficiently large (solid line in Fig. 61) the present theory predicts an additional 
reduction in the stress ratio occuring in the transitional regime, when both collisional and quasistatic stresses coexist. Here, the steady flow is characterized by the condition

$$
\frac{\tau}{\sigma}<\tan \phi_{s s}^{\prime}
$$

This peculiarity of the mechanical response will be discussed in detail in the next sections.

Fig. 7 shows the results of the present theory when the quasi-static contributions are ignored. The comparison of Fig. 7 with Fig. 6 allows to emphasize some key predictions of the theory: (i) the value of the concentration for $\dot{\gamma} \rightarrow 0$ would be independent on $\sigma$ in a purely collisional model; (ii) a purely collisional model cannot predict the asymptotic approach of the stress ratio to the critical friction angle for $\dot{\gamma} \rightarrow 0$ [14].

\subsection{Nonmonotonic behaviour in the transitional regime}

The condition for the occurence of a minimum for $\dot{\gamma} \neq 0$ and $\tau / \sigma<\tan \phi_{s s}^{\prime}$, in the transitional regime, (Fig. 6a) can be derived from Eq. (23). Indeed, for the shear rate being a real number,

$$
\frac{\tau / \sigma-\tan \phi_{s s}^{\prime}}{f_{4}\left(f_{2} f_{5}^{1 / 2}-\tan \phi_{s s}^{\prime} f_{1} f_{5}\right)}>0 .
$$

Hence, condition (31) can occur if

$$
f_{2} f_{5}^{1 / 2}-f_{1} f_{5} \tan \phi_{s s}^{\prime}<0
$$

given that $f_{4}$ is always positive. By using the expressions of Tab. [1, Eq. 33 gives

$$
\nu>\nu^{*}
$$

where

$$
\nu^{*}=\nu_{\mathrm{s}} \frac{\mathcal{B}^{9}\left(\tan \phi_{s s}^{\prime}\right)^{-9}}{\mathcal{B}^{9}\left(\tan \phi_{s s}^{\prime}\right)^{-9}+5.69\left(\nu_{\mathrm{s}}-0.49\right)},
$$

with

$$
\mathcal{B}=\left[\frac{48}{5 \pi(1+\epsilon)^{2}}\right]^{1 / 2}\left[\frac{\left(1-\epsilon^{2}\right)^{2} J^{4}}{15 c^{2}}\right]^{1 / 6},
$$

where $J$ and $F$ are calculated from Tab. [in the dense limit, that is, for $G \rightarrow \infty$ [26].

If $\nu_{\mathrm{m}}$ is larger than $\nu^{*}$, the material exibits a non-monotonic behaviour in the whole range of concentration between $\nu^{*}$ and $\nu_{\mathrm{m}}$. In contrast, if $\nu_{\mathrm{m}}<\nu^{*}$, the $\tau / \sigma$ trend is monotonically increasing. By using Eq. 30, the condition $\nu_{\mathrm{m}}>\nu^{*}$ corresponds to

$$
\sigma>\sigma^{*}
$$

where

$$
\sigma^{*}=a \frac{\left(\nu^{*}-\nu_{\mathrm{rlp}}\right)}{\left(\nu_{\mathrm{s}}-\nu^{*}\right)} \frac{K}{d} .
$$


The dependence of $\nu_{\mathrm{m}}$ on $\sigma$ and the values of $\nu$ and $\sigma$ satisfying conditions (34) and (37) are depicted in Fig. 8 ,

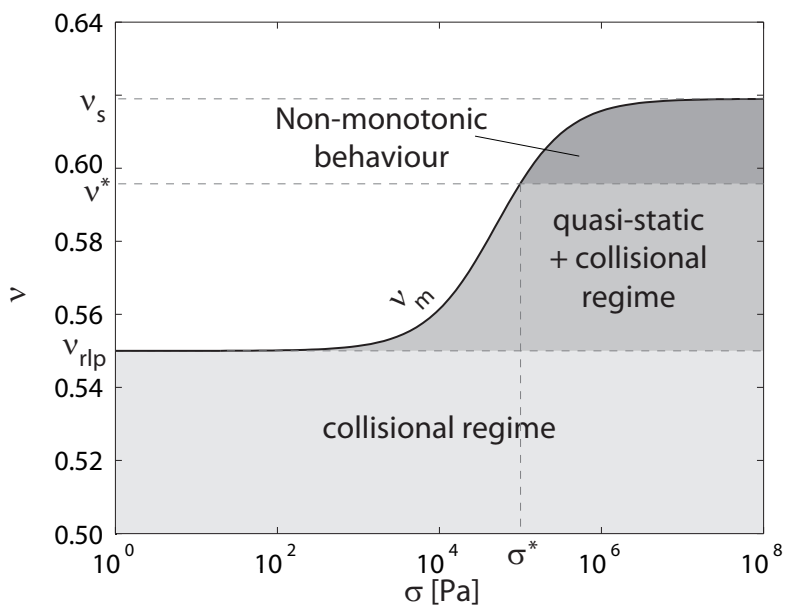

Figure 8. Maximum concentration attained in the steady, simple shear flow of $1 \mathrm{~mm}$ sand as function of the applied normal stress. The dark gray area represents the range of existence of the non monotonic behaviour for $\tau / \sigma$ when both collisional and quasi-static stresses coexist.

For $\nu>\nu^{*}$, the aforementioned visco-plastic interpretation previously suggested (Eq. 23) still holds, if the fluidity parameter is allowed to be an imaginary number. The dependence of the fluidity parameter on the concentration for different values of $\sigma$ is depicted in Fig. 9 . The gray area represents the range of concentration for which $\tilde{\gamma}$ is imaginary. This condition can not be a priori excluded but it must be further investigated by using either experimental or numerical tests. In fact, this unexpected trend could be a misleading consequence of the use of unphysical constitutive parameters as well as of having assumed $\epsilon$ to be constant, that is, independent on both $\sigma$ and the relative velocity among colliding particles.

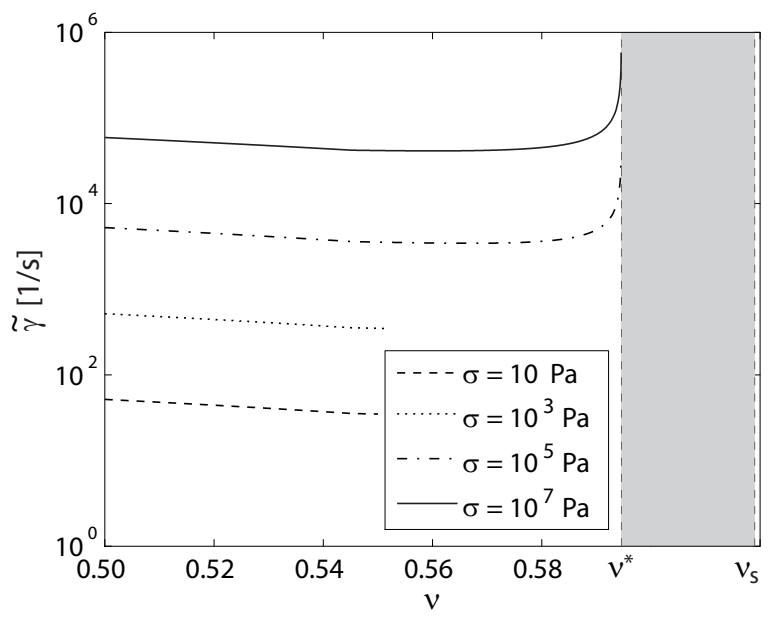

Figure 9. Fluidity parameter as a function of the concentration for different values of the normal stress. 


\subsection{Concentration ans stress ratio-imposed flows}

In order to highlight the nature of the constitutive relationship, and in particular the meaning of condition (31), or, equivalently, (34) and (37), in this section the steady state condition will be discussed with reference to (i) concentration and (ii) stress ratio-imposed flows. Here, all the graphs are obtained by employing the parameters previously defined.

(i) The concentration-imposed flow is a kinematic-control test where stresses are computed as a function of the two kinematic variables: concentration and shear rate. In this configuration, by using Eq. (20), the stress ratio is given by

$$
\frac{\tau}{\sigma}=\frac{\frac{K}{d} f_{0} \tan \phi_{s s}^{\prime}+\rho_{p} d^{2} f_{2} f_{4} f_{5}^{1 / 2} \dot{\gamma}^{2}}{\frac{K}{d} f_{0}+\rho_{p} d^{2} f_{1} f_{4} f_{5} \dot{\gamma}^{2}}
$$

where function $f_{4}$ is computed by substituting Eq. (17) into Eq. (11) as a function of the shear rate and of the imposed concentration.

Figures 10(a) and Fig. 10(b) show the results in terms of stress ratio versus shear rate and normal stress versus shear rate, respectively, for different values of the concentration.

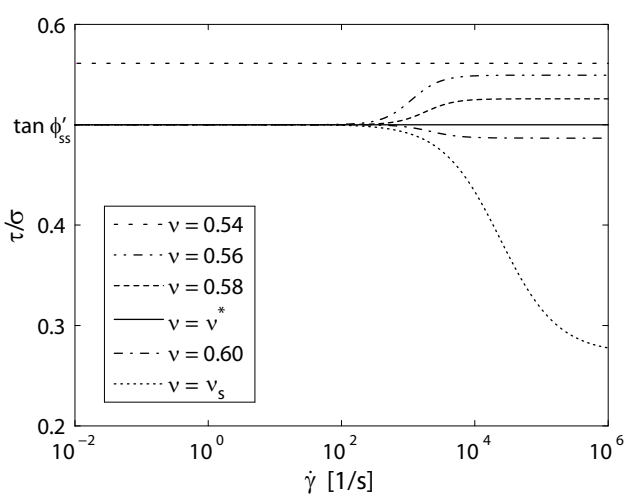

(a)

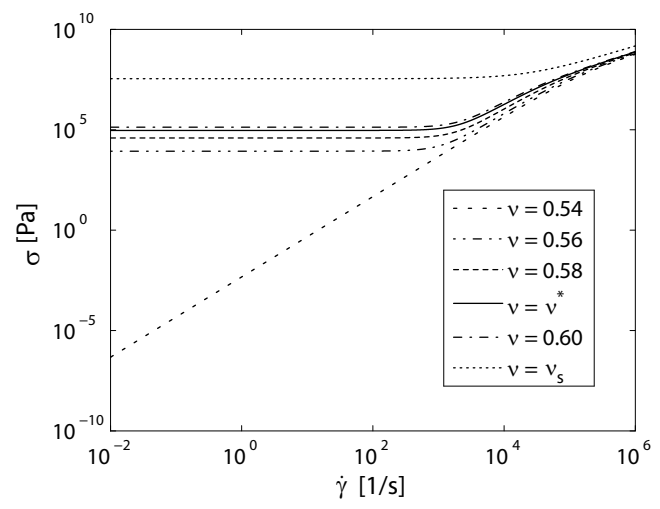

(b)

Figure 10. Theoretical (a) stress ratio and (b) normal stress as functions of the shear rate for $1 \mathrm{~mm}$ sand, at different values of the applied concentration.

Fig. 10(a) highlights the difference between the purely collisional and the transitional regime. Indeed, when the imposed concentration is lower than the random loose packing (collisional regime), the stress ratio is constant, as is predicted by the kinetic theory, whereas, in the transitional regime $\tau / \sigma$ is affected by the shear rate. The different shape of the normal stress in the two regimes is also evident in Fig. 10(b)

Furthermore, when the concentration is lower than $\nu^{*}$, the stress ratio is an increasing function of $\dot{\gamma}$, otherwise it presents a decreasing behaviour as a consequence of condition (34). The limit case is $\nu=\nu^{*}$, where the stress ratio is constant and equal to $\tan \phi_{s s}^{\prime}$ for all the values of the shear rate. 
(ii) A stress ratio-imposed flow is obtained by keeping constant the stress ratio $\tau / \sigma$ and measuring the shear rate and the concentration as a function of the normal stress.

In the transitional regime, i.e, when $\nu>\nu_{\text {rlp }}$, by using Eq. (39) and (20a), the relation between the concentration and the normal stress reads

$$
f_{0}\left[1+\frac{f_{1} f_{5}\left(\tan \phi_{s s}^{\prime}-\frac{\tau}{\sigma}\right)}{f_{1} f_{5} \frac{\tau}{\sigma}-f_{2} f_{5}^{1 / 2}}\right]=\frac{d}{K} \sigma
$$

and the shear rate is given by

$$
\dot{\gamma}=\sqrt{\frac{\sigma-\frac{K}{d} f_{0}}{\rho_{p} d^{2} f_{1} f_{4} f_{5}}},
$$

where $f_{4}$ is computed by using Eq. [15).

In the collisional regime $\left(\nu<\nu_{\text {rlp }}\right)$, the concentration is imposed by the stress ratio through

$$
\frac{f_{2}}{f_{1} f_{5}^{1 / 2}}=\frac{\tau}{\sigma}
$$

and it does not depend on the normal stress. Then, the shear rate reduces to

$$
\dot{\gamma}=\sqrt{\frac{\sigma}{\rho_{p} d^{2} f_{1} f_{4} f_{5}}} .
$$

The threshold between the two regimes is the stress ratio value associated with the random loose packing concentration, $(\tau / \sigma)_{\text {rlp }}$ (by employing the previously defined material parameters it is $0.558)$.

In Fig. 11(a) and 11(b) the concentration and the shear rate versus the normal stress, respectively, for different values of the imposed stress ratio, are plotted. The curves of Fig. 11(a) represent the iso-stress ratio lines in the phase diagram plane mentioned in Sect 3.1

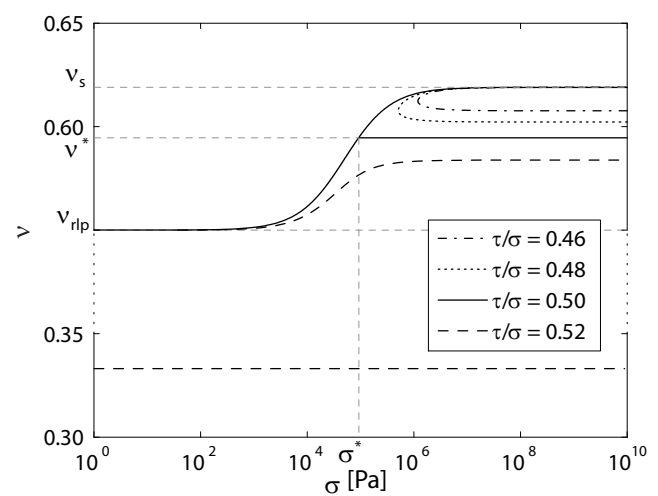

(a)

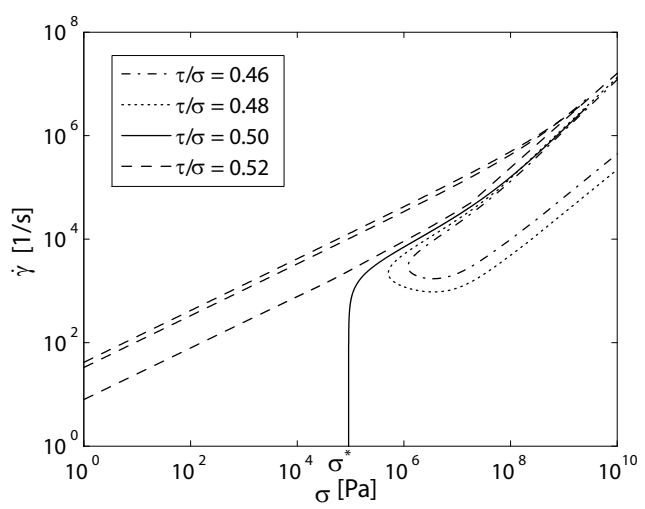

(b)

Figure 11. Theoretical (a) concentration and (b) shear rate as a function of the normal stress for $1 \mathrm{~mm}$ sand, at different values of the applied stress ratio. 
The two solid lines in Fig. 11(a) represent the limit case $\tau / \sigma=\tan \phi_{s s}^{\prime}=0.5$. From Eq. (39), by imposing $\tau / \sigma=\tan \phi_{s s}^{\prime}$, we obtain:

$$
\left(f_{2} f_{5}^{1 / 2}-f_{1} f_{5} \tan \phi_{s s}^{\prime}\right) \dot{\gamma}^{2}=0
$$

which leads to two solutions:

1. $\dot{\gamma}=0$ : it is the so called critical state, where the collisional contribution vanishes and the concentration is equal to $\nu_{\mathrm{m}}$ (30).

2. $f_{2} f_{5}^{1 / 2}-f_{1} f_{5} \tan \phi_{s s}^{\prime}=0$ : implying a concentration not depending on $\sigma$ and equal to $\nu^{*}$, as was stated in Eq. (35), and a shear rate defined, as usual, by Eq. (41).

When the normal stress reaches the $\sigma^{*}$ value (corresponding to $\nu_{\mathrm{m}}=\nu^{*}$ ), the steady flow undergoes a loss of uniqueness of the solution in terms of the two kinematic variables, $\nu$ and $\dot{\gamma}$.

This loss of uniqueness of the solution characterizes also all the curves at imposed stress ratio lower than $\tan \phi_{s s}^{\prime}$. Furthermore, when $\tau / \sigma<\tan \phi_{s s}^{\prime}$, there is only a limited range of the normal stress for which the steady state is possible, and, in this range, there are always two concentrations and two shear rates for a given value of $\sigma$. The range of existence of the steady state (range of possible normal stresses) increases accordingly to the imposed stress ratio, so the minimum normal stress moves on the left and tends to $\sigma^{*}$ as $\tau / \sigma$ tends to $\tan \phi_{s s}^{\prime}$. Then, all the curves characterized by an imposed stress ratio lower than $\tan \phi_{s s}^{\prime}$ are included between the two solid lines at $\tau / \sigma=\tan \phi_{s s}^{\prime}$, corresponding to the dark gray area of the phase diagram in Fig. 8 ,

Both the theoretical concentrations are larger than $\nu^{*}$, as was predicted by Eq. (34), and so both the steady states are in the transitional regime. In Fig. 12 a curve at applied stress ratio lower than $\tan \phi_{s s}^{\prime}$ is discussed.

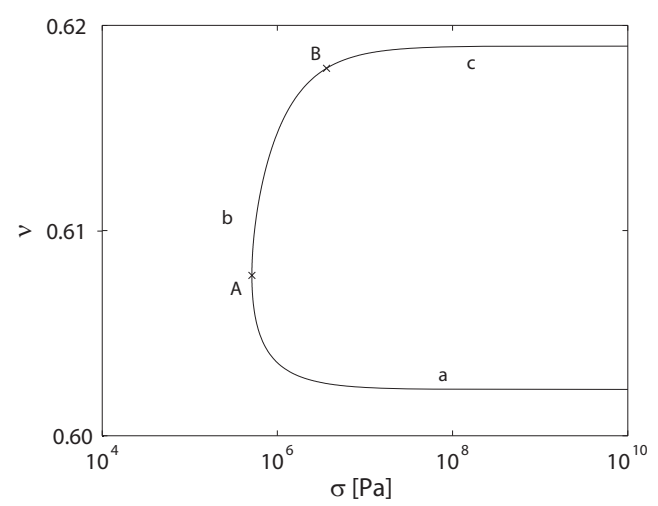

(a)

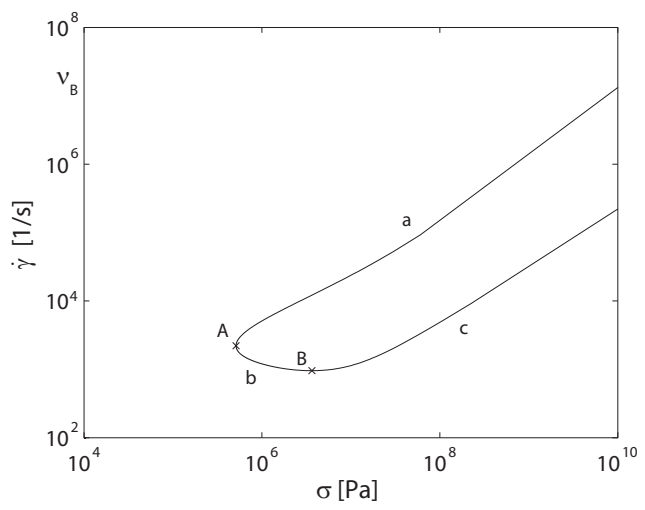

(b)

Figure 12. Theoretical (a) concentration and (b) shear rate as a function of the normal stress for $\tau / \sigma=0.48$.

Here, point $A$ corresponds with the minimum normal stress possible at the steady state, whereas $B$ represents the minimum shear rate achievable. Given these two points, we can distinguish three zones: 
zone a.: where the shear rate is an increasing function of the normal stress whereas concentration is deacresing;

zone b.: where the concentration increases and the shear rate decreases for increasing normal stresses;

zone c.: where both the shear rate and the concentration increase with the normal stress.

When the imposed stress ratio is greater than the tangent of the critical friction angle, $\tau / \sigma>\tan \phi_{s s}^{\prime}$, the steady state is possible for all values of $\sigma$. Furthermore, the loss of uniqueness of the solution can still occur depending on the value of $\tau / \sigma$. If the applied stress ratio is lower than $(\tau / \sigma)_{\text {rlp }}$, there is always a solution in the transitional regime, characterized by an increasing shear rate given by Eq. (41), and an increasing concentration (40), which varies with $\sigma$ between the random loose packing and the value $\nu^{*}$. Another solution can be found in the collisional regime. Here the concentration is constant as stated by Eq. (42), and the shear rate increases with the normal stress (43).

Given that, in the collisional regime, the minimum stress ratio, $(\tau / \sigma)_{\min }$, is reached for $\nu \approx 0.26$ and can be larger than $\tan \phi_{s s}^{\prime}$, there is a range of $\tau / \sigma$ where the steady state can not be reached in the collisional regime and the stress ratio-imposed flow solution is unique. For this particular ideal granular material, the minimum stress ratio in the collisional regime is $(\tau / \sigma)_{\min } \approx 0.513$, then for all the imposed stress ratio in the range $0.5-0.513$ the solution, in terms of $\nu$ and $\dot{\gamma}$, is unique and is in the transitional regime.

In contrast, when $(\tau / \sigma)_{\min }<\tau / \sigma<(\tau / \sigma)_{\text {rlp }}$, there is at least another concentration, lower than $\nu_{\mathrm{rlp}}$, and another shear rate solving the problem. The additional collisional solution can be one or more depending on the the imposed stress ratio since the kinetic theory predicts a non monotonic trend of the stress ratio versus concentration curve (Eq. 42). Referring to Fig. 11(b), for $\tau / \sigma$ equal to 0.52 there are three possible $\dot{\gamma}$, and also three concentrations, one in the transitional regime and two, constants, beneath $\nu_{\text {rlp }}$ (in Fig.11(a) only one of these two $\nu$ is plotted, the other one is lower than 0.3).

Finally, when the applied stress ratio is larger than the threshold $(\tau / \sigma)_{\text {rlp }}$, there are no possible solutions in the transitional regime, and the concentrations and shear rates solving the stress ratio-imposed flow have to be sought in the collisional regime (Eq.42,43).

It is worth noting that when stress ratio exceeds $\tan \phi_{s s}^{\prime}$ there is only one solution in the transitional regime and, if the loss of uniqueness occurs, the other solutions are in the purely collisional regime. On the other hand, the loss of uniqueness of the solution in the transitional regime is possible only if the imposed stress ratio is lower than $\tan \phi_{s s}^{\prime}$ (condition 31), corresponding to the dark gray area of Fig. 8 .

\section{CONCLUDING REMARKS}

This work has provided a theoretical framework, in which both standard Geotechnical constitutive models, based on the critical state theory, and kinetic theories of granular gases are merged. In particular, the steady state condition of a granular material under simple shear has been analyzed by employing a constitutive approach recently proposed by the authors, where both enduring contacts 
among particles involved in force chains and nearly instantaneous collisions are considered. The interpretation of the constitutive relationship in the light of standard visco-plasticity is a first step towards an evolving constitutive model capable of describing the mechanical behaviour of granular material under both solid-like and fluid-like conditions. Three flow configurations have been considered: pressure, concentration and stress ratio-imposed flows. Accounting for the stiffness of the particles allows to highlight the occurence of the limit condition $\tau / \sigma=\tan \phi_{s s}^{\prime}$, which produces a peculiar behaviour of the variable's profiles in the three considered flow conditions. Indeed, in the pressure-imposed flow, the stress ratio curves become non monotonic in the range of concentration between $\nu^{*}$ and $\nu_{\mathrm{m}}$, where the fluidity parameter gets imaginary. At the same time, in the concentration-imposed flow, the $\tau / \sigma$ function changes from an increasing to a decreasing dependence on the shear rate. Finally, the occurence of the same condition generates in the stress ratio-imposed flow a loss of uniqueness of the solution in the transitional regime, associated with a limited range of normal stresses at which steady state is possible. All the assumptions introduced to conceive the constitutive relationship (the restitution coefficient independent on both the normal stress and the relative velocity among colliding particles; the shear rigidity concentration independent on the friction coefficient; both the collisional and the quasi-static normal stresses diverging at the same concentration) have to be confirmed. This paper is thus to be interpreted as a theoretical stimulus for further experimental/numerical researches.

\section{REFERENCES}

1. Roscoe K, Schofield A, Wroth C. On the yielding of soils. Géotechnique 1958; 8(1):22-53.

2. Schofield A, Wroth C. Critical state soil mechanics. McGraw-Hill: London, U.K., 1968.

3. Been K, Jefferies M. Discussion: a state parameter for sands. Géotechnique 1986; 36(1):449-461.

4. Gajo A, Muir Wood D. Severn-Trent sand: a kinematic hardening constitutive model for sands: the $q-p$ formulation. Géotechnique 1999; 49(5):595-614.

5. Goldhirsch I. Rapid granular flows. Annual Review of Fluid Mechanics 2003; 35:267-293.

6. Jenkins J, Savage S. A theory for the rapid flow of identical, smooth, nearly elastic, spherical particles. Journal of Fluid Mechanics 1983; 130:187-202.

7. Savage S. The mechanics of rapid granular flows. Advances in Applied Mechanics 1984; $24: 289$.

8. di Prisco C, Pisanó F. Recenti sviluppi nell'analisi delle condizioni di innesco di movimenti franosi. Istituto Lombardo 2008; 142:147-170.

9. Berzi D, di Prisco C, Vescovi D. Constitutive relations for steady, dense granular flows. Physical Review E 2011; 84:031 301.

10. Abbott E. Flatland: A Romance of Many Dimensions. 1884.

11. Thornton C, Zhang L. Numerical simulations of the direct shear test. Chemical Engineering and Technology 2003; 26(2):153-156.

12. Bagnold R. Experiments on a gravity-free dispersion of large solid spheres in a newtonian fluid under shear. Proceedings of the Royal Society A 1954; 255(1160):49-63.

13. GDR-MiDi. On dense granular flows. European Physical Journal E 2004; 14:341-365.

14. da Cruz F, Emam S, Prochnow M, Roux J, Chevoir F. Rheophysics of dense granular materials: Discrete simulation of plane shear flows. Physical Review E 2005; 72:021 309.

15. Mitarai N, Nakanishi H. Velocity correlations in the dense granular shear flows: Effects on energy dissipation and normal stress. Physical Review E 2007; 75:031 305.

16. Johnson P, Jackson R. Frictional-collisional constitutive relations for granular materials, with application to plane shearing. Journal of Fluid Mechanics 1987; 176:67-93.

17. Johnson P, Jackson R. Frictional-collisional equations of motion for particulate flows and their application to chutes. Journal of Fluid Mechanics 1990; 210:501-535.

18. Savage S. Analyses of slow high-concentration flows of granulars. Journal of Fluid Mechanics 1998; 377:1-26. 
19. Lee C, Huang C. Model of sheared granular material and application to surface-driven granular flows under gravity. Physics of Fluids 2010; 22:043 307.

20. Muir Wood D. Geotechnical modelling. Spon Press: New York, 2004.

21. di Prisco C, Pisanó F. An exercise on slope stability and perfect elasto-plasticity. Géotechnique 2011; 61:923-934.

22. Ji S, Shen H. Internal parameters and regime map for soft polydispersed granular materials. Journal of Rheology 2008; 52:87-103.

23. Song C, Wang P, Makse H. A phase diagram for jammed matter. Nature 2008; 453:629-632.

24. Silbert L. Jamming of frictional spheres and random loose packing. Soft Matter 2010; 6(13):2918-2924.

25. Chialvo S, Sun J, Sundaresan S. Bridging the rheology of granular flows in three regimes. Physical Review E 2012; 85:021305.

26. Jenkins J, Berzi D. Dense inclined flows of inelastic spheres: tests of an extension of kinetic theory. Granular Matter 2010; 12(2):151-158.

27. Wroth C. Soil behaviour during shear - existence of critical void ratios. Engineering 1958; 186:409-413.

28. Muir Wood D. The magic of sands. Canadian Geotechnical Journal 2007; 44:1329-1350.

29. Garzó V, Dufty JW. Dense fluid transport for inelastic hard spheres. Physical Review E 1999; 59:5895.

30. Jenkins J, Zhang C. Kinetic theory for identical, frictional, nearly elastic spheres. Physics of Fluids 2002; 14(3): 1228.

31. Torquato S. Nearest-neighbor statistics for packing of hard spheres and disks. Physical Review E 1995; 51:3170.

32. Jenkins J. Dense shearing flows of inelastic disks. Physics of Fluids 2006; 18:103 307.

33. Jenkins J. Dense inclined flows of inelastic spheres. Granular Matter 2007; 10:47-52.

34. Hwang H, Hutter K. A new kinetic model for rapid granular flow. Continuum Mechanics and Thermodynamics 1995; 7:357-384.

35. Chapman S, Cowling T. The mathematical thoery of non-uniform gases. Cambridge University Press: Cambridge, U.K., 1970.

36. Perzyna P. Fundamental problems in viscoplasticity. Advances in Applied Mechanics 1966; 9:243-377.

37. Forterre Y, Pouliquen O. Long-surface-wave instability in dense granular flows. Journal of Fluid Mechanics 2003; 486:21-50. 